\title{
VON NEUMANN'S THEOREM ON ABELIAN FAMILIES OF OPERATORS
}

\section{B. R. GELBAUM}

The theorem referred to in the title is the following: If $\left\{A_{n}\right\}$ is a countable family of bounded commuting normal operators over an arbitrary Hilbert space (not necessarily separable), then there is a resolution of the identity $\{E(t) \mid 0 \leqq t \leqq 1\}$ and a sequence of continuous functions $\left\{a_{n}(t)\right\}$ such that, for all $n$,

$$
A_{n}=\int_{0}^{1} a_{n}(t) d E(t)
$$

The short proof below resembles but differs from von Neumann's original proof $[2 ; 3]$.

Let $\mathfrak{A}$ be the uniformly closed algebra generated by the set $\left\{I, A_{n}, A_{m}^{*}, n, m=1,2, \cdots\right\}$. Then, from the general theory of Banach algebras [1] we see that $\mathfrak{A}$ and $C(\mathfrak{M})$ are isometrically isomorphic and that the maximal ideal space $\mathfrak{M}$ of $\mathfrak{Q}$ is a compact metric space, since $\mathfrak{A}$ is separable. Hence there is a mapping $f: S \rightarrow \mathfrak{M}$ of the Cantor set $S$ onto $\mathfrak{M}$. For $t$ in $[0,1]$, let $\hat{E}_{t}(M)$ be the characteristic function of the set $f([0, t) \cap S) \subset \mathfrak{M}$. Each of these sets, as the union of the compact sets $f([0, t-1 / n] \cap S)$ is a Borel, hence since $\mathfrak{M}$ is metric, a Baire set.

In accordance with the isometric isomorphism between the set of bounded Baire functions on $\mathfrak{M}$ and a super-ring of $\mathfrak{A}[1,26 \mathrm{~F}, 26 \mathrm{G}]$, $\hat{E}_{t}(M)$ corresponds to a projection $E(t)$, and clearly $\{E(t) \mid 0 \leqq t \leqq 1\}$ is a resolution of the identity.

For $B$ in $\mathfrak{A}$, let $b(t)$ be defined as follows:

$$
b(t)=\left\{\begin{array}{l}
\hat{B}(f(t)), \quad t \in S ; \\
\alpha b\left(t_{1}\right)+\beta b\left(t_{2}\right), t=\alpha t_{1}+\beta t_{2}, \text { where }\left(t_{1}, t_{2}\right) \text { is one of the intervals } \\
\text { deleted in forming } S \text { and } 0 \leqq \alpha, \beta ; \alpha+\beta=1 .
\end{array}\right.
$$

Since $f$ and $\hat{B}$ are continuous, $b(t)$ is continuous. A direct computation shows $B=\int_{0}^{1} b(t) d E(t)$ and, in particular, $A_{n}=\int_{0}^{1} a_{n}(t) d E(t)$.

Indeed, for $\epsilon>0$, there is a $\delta>0$ such that if $\left|t_{1}-t_{2}\right|<\delta$ then $\left|b\left(t_{1}\right)-b\left(t_{2}\right)\right|<\epsilon$. Thus let $0=t_{0}<t_{1}<\cdots<t_{n}=1$ where $\max _{i}\left|t_{i+1}-t_{i}\right|<\delta$. For $\tau_{i} \in\left[t_{i}, t_{i+1}\right)$ we find 1963.

Received by the editors December 24, 1962 and, in revised form, February 13, 


$$
\begin{aligned}
\| B & -\sum_{i=0}^{n-1} b\left(\tau_{i}\right)\left[E\left(t_{i+1}\right)-E\left(t_{i}\right)\right] \| \\
& =\left\|\hat{B}(M)-\sum_{i=0}^{n-1} b\left(\tau_{i}\right)\left[\hat{E}_{t_{i+1}}(M)-\hat{E}_{t_{i}}(M)\right]\right\|_{\infty} .
\end{aligned}
$$

For any $M \in \mathfrak{M}$, there is a unique $i_{0}$ such that

$$
M \in f\left(\left[0, t_{i_{0}+1}\right) \cap S\right) \backslash f\left(\left[0, t_{i_{0}}\right) \cap S\right) .
$$

For this $M$, then, $\hat{E}_{t_{i+1}}(M)-\hat{E}_{t_{i}}(M)=0$ unless $i=i_{0}$, in which case $\hat{E}_{t_{i+1}}(M)-\hat{E}_{t_{i}}(M)=1$. Furthermore, $M=f(\tau)$, where $\tau \in\left(t_{i_{0}}, t_{i_{0}+1}\right) \cap S$. Thus $\hat{B}(M)=b(\tau)$ and

$$
\left|\hat{B}(M)-b\left(\tau_{i_{0}}\right)\right|=\left|b(\tau)-b\left(\tau_{i_{0}}\right)\right|<\epsilon .
$$

In short, $\left\|\hat{B}(M)-\sum_{i=0}^{n-2} b\left(\tau_{i}\right)\left[\hat{E}_{t_{i+1}}(M)-\hat{E}_{t_{i}}(M)\right]\right\|_{\infty}<\epsilon$, and finally $\left\|B-\sum_{i=0}^{n-1} b\left(\tau_{i}\right)\left[E\left(t_{i+1}\right)-E\left(t_{i}\right)\right]\right\|<\epsilon$. The required conclusion then follows.

The usual extensions of the above theorem to the cases where (a) the $A_{n}$ are not necessarily bounded or (b) $\left\{A_{n}\right\}$ is replaced by a not necessarily countable family $\left\{A_{\lambda}\right\}$ of not necessarily bounded, commuting normal operators on a separable Hilbert space, follow readily [2].

On the other hand, let $\mathfrak{a}$ be a cardinal greater than $2^{2 \mathrm{NoO}}$, and let $\Lambda=\{\lambda\}$ be a set of cardinality $a$. Then the set $l_{2}(\Lambda) \equiv\{x(\lambda) \mid x(\lambda)$ complex-valued, $\left.\sum_{\lambda \in \Lambda}|x(\lambda)|^{2}<\infty\right\}$ is a (highly nonseparable) Hilbert space on which the projections $P_{\mu}: x(\lambda) \rightarrow y(\lambda)=x(\mu) \delta_{\mu \lambda}$, form a commuting family of bounded Hermitian operators. If there were some resolution of the identity $\{E(t)\}$ such that for $\mu \in \Lambda$, $P_{\mu}=\int_{0}^{1} p_{\mu}(t) d E(t)$, where $p_{\mu}(t)$ is a complex-valued function, then the cardinality of the set $\left\{p_{\mu}(t)\right\}$ would be $a>2^{2 N_{0}}$, which is impossible, since the cardinality of the set of all complex-valued functions on $[0,1]$ is $2^{2 \mathrm{No}_{0}}$.

\section{BiBLIOGRAPHY}

1. L. Loomis, An introduction to abstract harmonic analysis, Van Nostrand, New York, 1953.

2. B. Sz.-Nagy, Spektraldarstellung linearer Transformationen des Hilbertschen Raumes, Springer, Berlin, 1942.

3. J. von Neumann, Zur Algebra der Funktionaloperationen und Theorie der normalen Operatoren, Math. Ann. 102 (1929), 370-427.

UNIVERSITY OF MINNESOTA 